
DR ARNAUD GREGOIRE L'HUILLIER (Orcid ID : 0000-0001-9230-7285)

DR YOICHIRO NATORI (Orcid ID : 0000-0002-4938-125X)

DR ELISA CORDERO (Orcid ID : 0000-0001-7766-7266)

DR DEEPALI KUMAR (Orcid ID : 0000-0003-1961-0477)

Article type : Original Article

\section{Natural Influenza Infection produces a greater Diversity of Humoral Responses than} Vaccination in Immunosuppressed Transplant Recipients

Cedric Hirzel (1, 2), Andrzej Chruscinski (3), Victor H. Ferreira (1), Arnaud G. L’Huillier (4), Yochiro Natori (5), Sang Hoon Han (6), Elisa Cordero (7,8), Atul Humar (1),

Deepali Kumar (1), Influenza in Transplant Study Group

(1) Transplant Infectious Diseases and Multi-Organ Transplant Program, University Health Network, Toronto, Ontario, Canada

(2) Department of Infectious Diseases, Bern University Hospital, University of Bern, Bern, Switzerland.

(3) Toronto General Hospital Research Institute, University Health Network, Toronto, Ontario, Canada.

(4) Pediatric Infectious Diseases Unit, Geneva University Hospitals and Faculty of Medicine, Geneva, Switzerland

(5) Division of Infectious Diseases, University of Miami, Miami, Florida, USA.

(6) University of South Korea, Seoul, South Korea

(7) Hospital Universitario Virgen del Rocío and Biomedicine Research Institute, Seville, Spain.

(8) Spanish Network for Research in Infectious Diseases (REIPI), Seville, Spain.

Cedric Hirzel and Andrzej Chruscinski share first authorship.

Atul Humar and Deepali Kumar share senior authorship.

This article has been accepted for publication and undergone full peer review but has not been through the copyediting, typesetting, pagination and proofreading process, which may lead to differences between this version and the Version of Record. Please cite this article as doi: $\underline{10.1111 / \text { AJT.16503 }}$

This article is protected by copyright. All rights reserved 


\section{Correspondence:}

Deepali Kumar

Email: deepali.kumar@uhn.ca

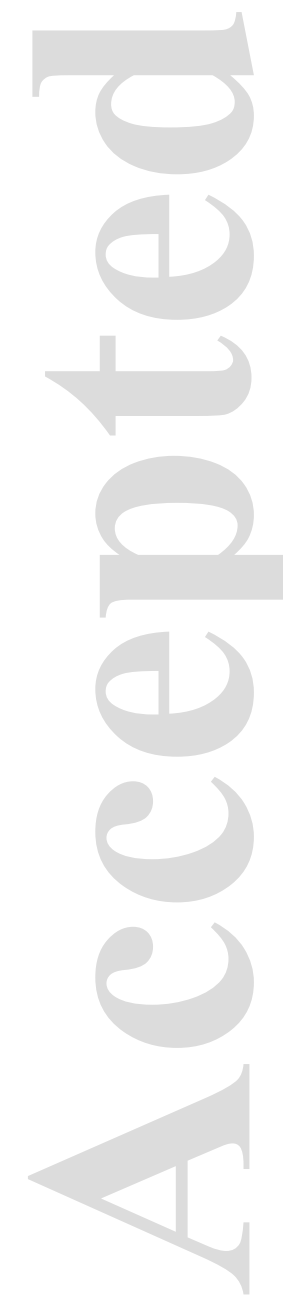

This article is protected by copyright. All rights reserved 


\section{ABBREVIATIONS:}

$\mathbf{C V}$, coefficient of variation

ELISA, enzyme linked immunosorbent assay

FBS, fetal bovine serum

HA1, hemagglutinin A1 subunit (hemagglutinin globular head protein)

HA2, hemagglutinin A2 subunit (hemagglutinin stem protein)

HI, hemagglutinin inhibiting

HIA, hemagglutinin inhibition assay

IAV, influenza A virus

IQR, interquartile range

MFI-B, median fluorescent intensity minus local background

M1, matrix 1 protein

NA, neuraminidase

NP, nucleoprotein

NS1, non-structural protein 1

NS2, non-structural protein 2

PA, polymerase acidic protein

PBS, phosphate-buffered saline

PB1, polymerase basic protein 1

PB2, polymerase basic protein 2

RNA, ribonucleic acid

SAM, significance analysis of microarray

SD, standard deviation

SOT, solid organ transplant 


\section{ABSTRACT}

The humoral immune response to influenza virus infection is complex and may be different compared to the antibody response elicited by vaccination. We analyzed the breadth of IgG and IgA responses in solid organ transplant recipients to a diverse collection of 86 influenza antigens elicited by natural influenza A virus (IAV) infection or by vaccination. Antibody levels were quantified using a custom antigen microarray. A total of 120 patients were included: 80 IAV infected (40 A/H1N1, $40 \mathrm{~A} / \mathrm{H} 3 \mathrm{~N} 2)$ and 40 vaccinated. Based on hierarchical clustering analysis, infection with either $\mathrm{H} 1 \mathrm{~N} 1$ or $\mathrm{H} 3 \mathrm{~N} 2$ virus showed a more diverse antibody response compared to vaccination. Similarly, H1N1 infected individuals showed a significant IgG response to $27.9 \%$ of array antigens, $\mathrm{H} 3 \mathrm{~N} 2$ infected patients to $43.0 \%$ of antigens, whereas vaccination elicited a less broad immune response ( $7.0 \%$ of antigens). Immune responses were not exclusively targeting influenza hemagglutinin proteins but were also directed against conserved influenza antigens. Serum IgA responses followed a similar profile. This study provides novel data on the breadth of antibody responses to influenza. We also found that the diversity of response is greater in influenza infected rather than vaccinated patients, providing a potential mechanistic rationale for suboptimal vaccine efficacy in this population. 


\section{INTRODUCTION}

Influenza virus infection causes significant morbidity and occasional mortality in the solid organ transplant (SOT) population ${ }^{1,2}$. A key aspect the immune response includes the development of antibodies directed against specific proteins within the influenza virus ${ }^{3-6}{ }^{7}$. Because of the immunosuppression required to avoid graft rejection, SOT recipients tend to have reduced antibody responses to both natural influenza infection and to vaccination ${ }^{8,9}$. In the vast majority of studies, vaccine response is measured primarily against the hemagglutinin protein of influenza. Based on this, transplant patients generally show variable but sub-optimal vaccine immunogenicity and they may still commonly acquire influenza despite vaccination. Less data are available on the humoral immune response to natural infection in transplantation but these also show a limited seroconversion when IgG antibodies are measured using a hemagglutination inhibition assay ${ }^{8}$.

Influenza virus is a member of the Orthomyxoviridae family. The influenza A (IAV) viral genome consists of eight negative-sense single stranded RNA fragments, which code for different viral proteins, including hemagglutinin (HA), neuraminidase (NA), matrix protein (M1 and M2), polymerase protein subunits (PB1, PB2, PA), nucleoprotein (NP), and non-structural proteins (NS1, NS2) ${ }^{10}$. The two major transmembrane glycoproteins of the virus, HA and NA, show continuously shifting amino acid sequences and are therefore antigenically highly variable ${ }^{11}$. Other parts of human pathogenic IAVs, such as the external domain of the M2 protein $(\sim 90 \%$ conserved among influenza A viruses), NP ( $~ 90 \%$ conserved), M1 ( $>90 \%$ conserved), NS1 ( $>90 \%$ conserved), NS2 ( $90 \%$ conserved) are much less variable ${ }^{11-15}$. Traditionally humoral immune responses to infection and vaccination against hemagglutinins have been studied extensively because these antibodies (hemagglutinin inhibiting antibodies) correlate with protection from infection ${ }^{16}$. However, influenza vaccination or infection may also elicit antibody responses to influenza proteins other than strain-specific HA proteins ${ }^{11}$. For instance, mouse studies show that the presence of anti-NA antibodies was correlated with milder disease and anti-M2 antibodies can reduce influenza viral loads and are associated with better survival after viral challenge ${ }^{11,16}$.

Antigen microarrays can be a useful tool for serologic profiling of humoral immune responses to a range of influenza antigens ${ }^{17-22}$. The array technique (as opposed to ELISA) allows for multiplex testing against many different influenza antigens using only small volumes of serum. Two-color detection protocols can also be used to simultaneously measure two antibody classes (e.g. IgG and IgA) on the same slide surface. 
There are limited data on the diversity of influenza-specific antibody responses in SOT recipients. We hypothesized that natural infection would likely exhibit a much more robust and diverse antibody response as compared to influenza vaccination. This would partly help explain the suboptimal vaccine efficacy observed in transplant patients. It would also be important to understand the development of antibodies against conserved viral epitopes, and heterologous strain proteins, to help delineate how both vaccination and infection may provide broader protection against other influenza strains. Therefore, the aim of our study was to investigate the diversity of anti-influenza antibody responses to natural infection and vaccination in SOT recipients and to compare differences in humoral immune responses after vaccination and natural infection. 


\section{METHODS}

\subsection{Patients and Sample Collection}

Two groups of adult (aged $\geq 18$ years) solid organ transplant (SOT) recipients were included in this study: 1) patients with IAV infection and 2) patients who were vaccinated with one dose of inactivated trivalent influenza vaccine.

Serum samples from IAV infected SOT recipients (40 patients with influenza A/H1N1 infection and 40 patients with influenza $\mathrm{A} / \mathrm{H} 3 \mathrm{~N} 2$ infection) were obtained from a prospective multicenter cohort study conducted at 20 different centers in the United States, Canada and Spain during the 2010-2015 influenza seasons ${ }^{1}$. Samples were chosen if they had an adequate amount of serum at two timepoints. All patients had laboratory confirmed (by center specific nucleic acid amplification testing) diagnosis of influenza A infection. Serum samples were drawn at enrollment and four weeks after.

Serum samples from vaccinated patients $(n=40)$ were obtained from a double-blind randomized controlled influenza vaccine trial ${ }^{23}$. Similar to the infected patients, samples with enough serum to perform the study were chosen. Patients were vaccinated with the 2016-17 trivalent influenza vaccine Fluviral ${ }^{\circledR}$ (GSK, Canada), which is a split-virus vaccine. The vaccine contained antigens (15 $\mu \mathrm{g}$ per antigen) of the following three viruses: A/California/7/2009 (H1N1)pdm09-like virus, A/Hong Kong/4801/2014 (H3N2)-like virus, and B/Brisbane/60/2008like virus. Sera were drawn immediately before and four weeks after vaccination.

\subsection{Antigen Library}

A diverse collection of influenza antigens $(n=86)$, including those from various subtypes (influenza A/H1N1, A/H3N2, A/H5N1, influenza B) and geographical locations were used. The microarray consisted of $26 \mathrm{~A} / \mathrm{H} 1 \mathrm{~N} 1$ antigens (14 targeting HA, $4 \mathrm{NA}$, and 8 targeting conserved antigens), $35 \mathrm{~A} / \mathrm{H} 3 \mathrm{~N} 2$ antigens (27 targeting HA, 2 NA, 2 whole viral particles, and 3 targeting conserved antigens), $6 \mathrm{~A} / \mathrm{H} 5 \mathrm{~N} 1$ antigens ( 3 targeting HA, $1 \mathrm{NA}$, and 2 conserved antigens), and 19 influenza B antigens (14 targeting HA, 1 NA, 3 whole viral particles, and 2 conserved antigens). A complete list of all antigens arrayed is provided in the Supporting information (Supplementary Table 1). Antigens were diluted to $0.2 \mathrm{mg} / \mathrm{ml}$ in PBS and stored in aliquots at $80^{\circ} \mathrm{C}$ until the day of microarray printing.

\subsection{Antigen Microarrays}

The influenza antigen microarray was generated using previously published protocols for generation of antigen microarrays to screen for autoantibodies in heart failure and transplantation 
24-26. Antigens including whole virus lysates, proteins and peptides were spotted in duplicate onto two-pad nitrocellulose coated slides (Oncocyte ${ }^{\circledR}$ SuperNOVA, Grace Bio-Labs, Bend, OR, USA) using a VersArray Chipwriter Pro microarrayer (Virtek Vision, Waterloo, ON, Canada). Slides were arrayed at room temperature at a relative humidity of 55\%. Solid pins (Arrayit, Sunnyvale, CA, USA) were used to generate features of approximately $500 \mu \mathrm{m}$ in diameter. Dried slides were placed in FAST frames (Maine Manufacturing, Sanford, ME, USA) and blocked overnight at $4{ }^{\circ} \mathrm{C}$ (blocking buffer: PBS, 5\% FBS, 0.1\% Tween). The next day, arrays were incubated with patient serum (diluted 1:100 in blocking buffer) for one hour at $4^{\circ} \mathrm{C}$. Baseline and convalescent (or postvaccination) serum from same patient were tested on separate pads from on same slide. Thereafter slides were washed extensively (PBS with $0.1 \%$ Tween) and probed and incubated for 45 minutes at $4{ }^{\circ} \mathrm{C}$ with a mixture of secondary antibodies consisting of Cy3-labeled goat anti-human IgG (Jackson ImmunoResearch, West Grove, PA, USA) at a dilution of 1:2000 and Alexa Fluor 647labeled goat anti-human IgA (Jackson ImmunoResearch, West Grove, PA, USA) at a dilution of 1:1000. After washing, slides were dried by centrifugation ( $220 \mathrm{x} g$ for 5 minutes).

Fluorescent intensities of features were quantified using an Axon 4200A microarray scanner (Molecular Devices, Sunnyvale, CA, USA) and Genepix 6.1. software (Molecular Devices, San Jose, CA, USA). Median fluorescent intensity minus local background (MFI-B) was determined (at $532 \mathrm{~nm}$ for Cy3 and $635 \mathrm{~nm}$ for Alexa Fluor 647). The single averaged MFI-B for each antigen was calculated from the features arrayed in duplicate (for illustration of the antigen microarray see Supplementary Figure 1).

\subsection{Quality Control}

The interslide variation (variability of signal within arrays tested the same day) was determined by measuring the variation coefficient of 7 slides (14 nitrocellulose pads) which were probed with the same serum sample and processed the same day. The mean CV $( \pm 1 \mathrm{SD})$ was $7.6 \%( \pm 3.9)$ for $\operatorname{IgG}$ signals and $15.2 \%( \pm 10.7)$ for IgA signals. Linearity of the assay was assessed by plotting $\log 2-$ transformed signals for the different antigens from serially diluted sera (example shown in Supplementary Figures 2 and 3).

To reduce any impact of signal variability in the analysis, we decided to compare foldchanges in MFI-B (MFI-B after 4 weeks / MFI-B at baseline) instead of focusing on absolute MFI-B values. This was possible because of the linearity of the assay and the fact that baseline and 4-week serum samples of the same patient were probed on the same slide. Seroconversion was defined as a $\geq 4$-fold increase in MFI-B. 


\subsection{Hemagglutination inhibition assay (HIA)}

For validation of array against a standardized HIA, hemagglutinin inhibiting (HI) antibody titers for H1N1 A/California/07/2009 were determined for all 120 samples used in the study. HIAs were performed at a World Health Organization (WHO) national influenza reference laboratory (Public Health England) using the methods described previously ${ }^{27}$. Titers were determined in duplicate by doubling dilutions of serum using an initial dilution of 1:10. Antibody concentrations below the lower limit of detection $(<1: 10)$ were assigned a titer of 5 for the purposes of analysis.

Seroconversion was defined as $\mathrm{a} \geq 4$-fold increase in HI antibody titer.

\subsection{Statistical Analysis}

Demographic characteristics between IAV infected SOT recipients and vaccinated patients were compared using descriptive statistics (Chi-squared test or Fisher`s exact test for categorical variables; Mann-Whitney-U test for continuous variables).

Differences in antigen reactivity between baseline and 4-week serum samples were identified as follows: we explored if $\log 2$ transformed MFI-B fold changes were different from zero by using Significance Analysis of Microarrays (SAM) with a false discovery rate of $<1 \%$ (q-value $<0.01$ ) 28. Only antigen reactivities with a q-value of $<0.01$ that changed at least 2 -fold were considered to be significant.

In order to compare antibody responses of $\mathrm{A} / \mathrm{H} 1 \mathrm{~N} 1$ infected vs. vaccinated patients against $\mathrm{H} 1 \mathrm{~N} 1$ antigens, $\log 2$ transformed MFI-B fold changes of $\mathrm{A} / \mathrm{H} 1 \mathrm{~N} 1$ antigen reactivities were compared using SAM with a false discovery rate of $1 \%$ (q-value $<0.01$ ). The same approach was applied for A/H3N2 infected vs. vaccinated patients. Following SAM analysis, hierarchical clustering (Manhattan clustering) of significantly different antigen reactivities was performed using Cluster 3.0 and heatmaps were generated with Treeview $1.60^{29}$. SAM analysis was performed using an Excel add-in ${ }^{28}$. Descriptive statistics were done using Stata software version 12.0 (College Station, TX, USA). 


\section{RESULTS}

\subsection{Cohort Description}

A total of 120 patients were analyzed. Baseline demographics are shown in Table 1. Almost half of the cohort were kidney transplant recipients (48.3\%). Kidney transplant recipients were more represented in the IAV infected population (57.5\%) compared to the vaccine group $(30.0 \%)$; $\mathrm{p}=0.006$. The proportion of the other organ types between the two groups was not statistically different although there was a trend to a higher proportion of heart or lung transplant patients in the vaccine group. However, the overall immunosuppression regimen was very similar across groups. Most patients were on calcineurin inhibitor-based immunosuppressive regimens (95.0\%); six patients had combined calcineurin-inhibitor/sirolimus immunosuppression. All IAV infected transplant recipients were treated with oseltamivir.

\subsection{Antibody response to influenza infection and vaccination}

Antibody response in influenza $\mathrm{A} / \mathrm{H} 1 \mathrm{~N} 1$ infected transplant patients

Of the 86 antigens coated on the microarray, 26 were influenza A/H1N1 antigens (Supplementary Table 1), including 14 complete HA proteins and 8 peptides corresponding to the globular head of HA (HA1). When averaged across all 40 patients, Influenza A/H1N1 infection resulted in a significant IgG immune response (mean of $>2$-fold change in fluorescence) against several HA antigens from different strains of H1N1 (Figure 1 and Supplementary Table 2) as well as other antigens. A total of $27.9 \%(24 / 86)$ of the antigens included in the microarray (Figure 1A) showed a mean 2-fold or higher change in reactivity across the 40 patients. Of these, the immune response was mainly directed against influenza A/H1N1 antigens $(75.0 \% ; 18 / 24)$. However, heterosubtypic immune responses targeting six H3N2 or H5N1 antigens were also identified. Of note, 5 out of these 6 antigens were either matrix 1 (M1), nucleoproteins (NP), or a hemagglutinin stem protein (HA2) (Supplementary Table 2). All these proteins are considered well-conserved influenza A proteins ${ }^{11}$.

Significant IgA responses were identified against 23.3\% (20/86) of the antigens (Figure 1B). Again, the immune response was mostly directed against influenza $\mathrm{A} / \mathrm{H} 1 \mathrm{~N} 1$ antigens $(80.0 \%$; 16/20) (Supplementary Table 2). The majority $(75.0 \% ; 3 / 4)$ of the significant IgA antibody responses against non-A/H1N1 antigens was directed against conserved influenza A antigens (two M1 proteins and a HA2 protein).All antigens with a significant IgA response were also significant for IgG antibody response. However, 16.7\% (4/24) of antigens for which we found significant IgG immune responses did not show IgA antibody responses (Supplementary Table 2). 
Antibody response in influenza A/H3N2 infected transplant patients

The antigen microarray included 35 different influenza A/H3N2 antigens (including 11 complete HA proteins, and 15 HA1 peptides; Supplementary Table 1). When averaged across all 40 patients with H3N2 infection, a significant IgG response was detected against $43.0 \%(37 / 86)$ of the antigens included in the microarray (Figure 1A). The antibody response was mainly targeting A/H3N2 antigens $(83.8 \% ; 33 / 37)$ (Supplementary Table 3). Heterosubtypic responses to six nonA/H3N2 antigens were present, and mainly targeted conserved IAV antigens including three M1 proteins, one NP protein and one NS1 protein (Supplementary Table 3).

IgA antibody responses were present for 37.2\% (32/86) of the antigens (Figure 1B). The IgA response was primarily detectable against A/H3N2 antigens $(81.3 \%, 26 / 32)$ (Supplementary Table 3). Most of the antigens against which we detected significant IgA antibody responses also showed significant IgG responses $(90.6 \% ; 29 / 32)$. However, for $21.6 \%(8 / 37)$ of antigens with significant IgG immune responses there was no significant IgA immune response (Supplementary Table 3).

Antibody response to trivalent influenza vaccine

The diversity of antibody responses in vaccinated transplant recipients was poor. When averaged across 40 patients, significant IgG antibody responses were observed to only $7.0 \%(6 / 86)$ of the antigens (Figure 1A). The vaccine contains H1N1, H3N2, and B viruses (and was a split virus vaccine preparation). However, responses were only observed against influenza A virus and predominantly H1N1 antigens (5/6 83.3\%). Three of the responses were against the H1N1 HA1 protein (the globular head) while three were against the M1 protein (2 H1N1 and $1 \mathrm{H} 3 \mathrm{~N} 2$ ). (Supplementary Table 4).

Significant IgA responses were detected for 5.8\% (5/86) of the antigens (Figure 1B). These antigens were either complete HA proteins or the HA1 globular head protein (Supplementary Table 4). Three out of five antigens $(60.0 \%)$ with significant IgA responses had also significant IgG antibody responses. For half $(3 / 6 ; 50.0 \%)$ of antigens with $\mathrm{IgG}$ immune responses there were no significant IgA antibody production (Supplementary Table 4). Of note, the overall median IgG MFI's of the post-vaccination sera (median: 1926.0, IQR: 1033.0-2638.5) were 7.9 times higher compared to the IgA signal intensity (median: 243.6, IQR: 164.3-500.3, p<0.0001).

\subsection{Differences in antibody responses to influenza $A$ infection versus vaccination}

Influenza $\mathrm{A} / \mathrm{H} 1 \mathrm{~N} 1 \mathrm{pdm}$ infection versus vaccination 
A visual representation of microarray responses ( $\mathrm{H} 1 \mathrm{~N} 1$ natural infection and vaccination) is shown in Figure 2, which demonstrates a clear distinction between patients with natural infection vs. vaccination. In order to determine differences in fold changes of $\mathrm{IgG}$ antigen reactivity between influenza $\mathrm{A} / \mathrm{H} 1 \mathrm{~N} 1$ infected and vaccinated SOT recipients, SAM analysis was performed, which revealed $13 \mathrm{~A} / \mathrm{H} 1 \mathrm{~N} 1$ antigen reactivities to be significantly (q-values $<0.01$ ) different in the influenza $\mathrm{A} / \mathrm{H} 1 \mathrm{~N} 1$ infected group versus the vaccinated group. Hierarchical clustering of these antigens revealed that patients with high IgG responses derived exclusively from the infected group (i.e. all 13 differentially expressed antigen reactivities were higher in the natural infection group vs. the vaccine group) (Figure 2). Similarly, SAM analysis identified $11 \mathrm{~A} / \mathrm{H} 1 \mathrm{~N} 1$ antigen IgA reactivities which were significantly (q-values $<0.01)$ different in the influenza $\mathrm{A} / \mathrm{H} 1 \mathrm{~N} 1$ infected group compared to the vaccine group (Supplementary Figure 4). Clustering of the IgA reactivities was less clear. However, two main clusters were detected and most (11/13) patients in the cluster with high IgA responses represented patients with $\mathrm{A} / \mathrm{H} 1 \mathrm{~N} 1$ infection.

Patients who were naturally infected despite having had an influenza vaccine within the same influenza season (24/40 H1N1 infected patients) did not show a differing response pattern than unvaccinated patients (Figure 2, Supplementary Figure 4).

Influenza A/H3N2 infection versus vaccination

A visual representation of microarray responses ( $\mathrm{H} 3 \mathrm{~N} 2$ natural infection and vaccination) is shown in Figure 3, which again shows a clear distinction between patients with infection vs. vaccination. SAM analysis identified 18 significantly different $\mathrm{A} / \mathrm{H} 3 \mathrm{~N} 2 \mathrm{IgG}$ antigen reactivities among $\mathrm{A} / \mathrm{H} 3 \mathrm{~N} 2$ infected and vaccinated SOT patients. Hierarchical clustering demonstrated that infected patients represented the vast majority $(25 / 27 ; 92.6 \%)$ of the patients who clustered in the high responder group while vaccinated patients clustered primarily in the low responder group. (Figure 3). Similarly, for IgA, compared to vaccination, influenza A/H3N2 infection resulted in significantly (q-values $<0.01$ ) higher fold changes in reactivity against 17 antigens (Supplementary Figure 5). Again, all these antigens were either complete HA proteins or HA peptides.

Despite receiving influenza vaccine within the same influenza season (28/40 H3N2 infected patients) did not show a distinct response pattern (Figure 3, Supplementary Figure 5). 3.4 Seroconversion for H1N1 A/California/07/2009 hemagglutinin antibodies by HIA and microarray 
In order to determine the concordance between seroconversion ( $\geq 4$-fold rise from pre-vaccination to post-vaccination in vaccinated patients or onset of illness vs. convalescence in infected patients) and $\geq 4$-fold rise in MFI-B, all sera underwent HIA for H1N1 A/California/07/2009. This specific antigen was chosen since it was circulating during the 2010-2015 influenza seasons. It was also included in the 2016-17 vaccine. Using this antigen, the inter-test reliability of HIA and microarray for seroconversion was $85.0 \%$ (concordant results for 102/120 tests)(Supplementary Table 5). 


\section{DISCUSSION}

Using custom antigen microarrays, we profiled the diversity of antibody responses in immunosuppressed transplant cohorts of patients infected with influenza A/H1N1 and A/H3N2 and compared them to vaccinated transplant recipients. This led to a number of novel findings. We found that natural infection elicits a relatively robust and diverse antibody response. These antibodies are primarily directed towards the antigens of the corresponding influenza A subtype and mainly to either the whole hemagglutinin protein or a subunit. However, a proportion of patients also showed an increase in antibodies to conserved antigens (such as M1, NP, and NS1) as well as to heterosubtypic antigens (i.e. antigens from other strains of influenza). We also showed that the diversity of antibody response is substantially greater in infected rather than vaccinated patients. This was evident in the absolute numbers of antigens that elicited an overall response as well as in a comparative clustering analysis of individual antigen reactivities in vaccinated vs. infected patients. We believe this provides unique insight into antigen responsiveness in the setting of immunosuppression, and lays a better foundation to develop strategies to improve vaccine immunogenicity in this patient population.

There are limited data on the diversity of antibody responses to influenza in transplant patients. However, studies in immunocompetent patients suggests that the majority of antibodies induced by natural influenza infection will target HA proteins and that there will be lower-level responses to NA and internal, more conserved, viral proteins ${ }^{30}$. The breadth of the response to HAs induced by influenza infection mainly depends on the exposure and vaccination history of the infected individual. This phenomenon, also known as the original antigenic sin, describes the increases in antibody titers to historic strains that can occur after immunization or infection with a new influenza strain ${ }^{30-32}$. This may explain why we found some significant antibody responses to older, currently not circulating HA proteins, included in our assay. Antibodies towards the NA protein are also induced in healthy individuals by natural infection ${ }^{30}$. However, NA antibody responses are typically lower compared to hemagglutinin responses. In addition, N1 neuraminidase proteins seem to be less immunogenic than N2 neuraminidase proteins ${ }^{33}$. In agreement with these findings, we detected anti-N2 neuraminidase immune responses in influenza A/H3N2 infected SOT recipients, but we did not find significant immune responses against N1 neuraminidases in our A/H1N1 infected patients. Antibodies to NP have also been previously reported after natural infection in healthy individuals ${ }^{34,35}$. Consistent with the findings in immunocompetent persons, we also detected anti-M1 antibodies after natural IAV infection in our 
immunocompromised population ${ }^{36,37}$. We also noted that in a small minority of patients, MFI-B values decreased from the first to second sample. It is possible that depending on symptom onset, we may have missed the peak of the antibody response. Alternatively, augmentation of immunosuppression between the first and second serum collections may have had some impact on a small subset of patients.

Split virus vaccines, such as the vaccine used in our study, are manufactured using inactivated influenza viruses, which are treated with a detergent and further purified. Only the content of the HA proteins in these vaccines is standardized, and the content of the other proteins is likely to depend on the specific vaccine or even the batch of the vaccine, but such proteins are present ${ }^{30}$. Therefore, antibodies against non-HA influenza proteins can also be induced by vaccination. In the immunocompetent population antibody responses against M1, NP, and NA proteins have been found thus far ${ }^{34,38,39}$. However, compared with natural infection the response induced by vaccination with a split virus vaccine is relatively narrow in immunocompetent persons ${ }^{30}$. In our study, we can confirm that these findings also apply for the immunocompromised population. But we would like to highlight that influenza vaccination in transplant recipients usually generates lower antigen-specific antibody responses in comparison to the general population due to exogenous immunosuppression. Despite this, there are no antibody cut-offs that clearly define protection from disease, although a titer of 1:40 is commonly used for vaccine-associated seroprotection. Although we find the antibody titers as noted by microarray to be relatively lower for vaccinated patients, this does not imply lack of clinical protection. We have previously shown that in a multicenter cohort of transplant patients infected with influenza, prior vaccination in the same influenza season protects patients from severe illness ${ }^{1}$. In addition, the specific vaccine used in the study was the standard-dose $(15 \mu \mathrm{g})$ trivalent influenza vaccine. A previous randomized trial showed that high-dose influenza vaccine $(60 \mu \mathrm{g} /$ antigen) elicited a significantly greater humoral response in organ transplant recipients vs. standard-dose vaccine ${ }^{40}$. A number of different approaches have the potential to improve and broaden influenza vaccine responses in transplant recipients including high-dose vaccines, MF59-adjuvanted vaccine and recombinant influenza vaccines. The ability of these vaccines to elicit a greater breadth of antibodies as detected by antigen microarrays will be investigated in future studies.

Another novel aspect of this study was the measurement of IgA responses. Indeed, we found that a serum IgA antibody response is induced upon influenza A infection and also with vaccination. The responses generally correlated with $\mathrm{IgG}$ responses in terms of antigen reactivity. 
In human serum, IgG constitutes approximately $75 \%$ and $\operatorname{IgA} 15 \%$ of antibody ${ }^{41}$. Both, serum IgG and serum IgA antibody responses (measured by ELISA) to natural IAV infection and to intramuscular vaccination with an inactivated IAV vaccine have been reported previously in nonimmunocompromised individuals ${ }^{42-44}$. The role of viral specific serum IgA for protection against influenza or viral clearance is unclear. However, the results of a human influenza challenge study in healthy volunteers suggests that there is a weak inverse correlation between influenza specific serum IgA antibody concentration and viral shedding measured by culture ${ }^{44}$.

One limitation of our study is that our analysis was that we were restricted to antigens that were commercially available. These may not have exactly matched the antigens in circulating influenza strains or vaccine strains although we attempted to have a diversity of antigens. In addition, the antigens used in our assay were produced by several different manufacturers using varying expression systems. This may have negatively affected the detection for some of the antibody responses because the microarray technology does not allow to optimize the assay for each individual antigen. Another limitation is that there is no specific cut-off MFI that implies protection. We addressed this by comparing fold-changes in MFI-B instead of focusing on absolute values ${ }^{45}$. It is possible that conformational epitopes may not be properly displayed for antibody binding; however, studies using the same platform for autoimmune antigens have shown correlation between traditional ELISA and antigen microarrays ${ }^{24,46}$. We also found a concordance of $85 \%$ between our microarray technique and HIA for one of the common influenza strains during the study period. The design of the study also meant that only medically attended cases of influenza were enrolled. This means that mildly symptomatic cases that may perhaps have a different antibody response profile after infection could be underrepresented. Furthermore, there was a slight overrepresentation of kidney transplant recipients in the naturally infected group compared to the vaccinated population. As the immunosuppressive regimens among the two groups were comparable, we do not believe that this had a major impact on our findings although it is not possible to exclude the impact of more granular differences in immunosuppresion. Nontransplant controls were not included in this study and this is a limitation. Therefore, study results need to be interpreted in this context.

In summary, we provide novel information profiling the diversity of antibody response to natural influenza infection and vaccination in the transplant population. We show that natural infection resulted in a substantially broader humoral immune response compared to vaccination with a split virus vaccine. Thus, vaccines with greater antigenic diversity may better mimic the 
immune responses elicited by natural infection. We also provide novel data on response to conserved virus antigens. A better understanding of the differences between natural infection and vaccination will likely be helpful to design better vaccines for immunocompromised patients. 


\section{DATA AVAILABILITY STATEMENT}

The data that support the findings of this study are available from the corresponding author upon reasonable request.

\section{ACKNOWLEDGMENTS}

C.H. was supported by an early Postdoc.Mobility grant from the Swiss National Science Foundation (P2BEP3_175265). A.L. was supported by an advanced Postdoc. Mobility grant from the Swiss National Science Foundation (P300PB_171603).

\section{DISCLOSURE}

The authors of this manuscript have conflicts of interest to disclose as described by the American Journal of Transplantation. D. K. has received research grants from Roche and GSK, and honoraria from GSK, Roche, Sanofi and Merck. A. H. has received a research grant from Roche. V.H. F. has received a travel grant from Roche. The other authors have no conflict of interest to disclose.

\section{ORCID ID:}

Cedric Hirzel, 0000-0002-7870-912X; Yoichiro Natori, 0000-0002-4938-125X;

Deepali Kumar, 0000-0003-1961-0477

\section{AUTHOR CONTRIBUTIONS:}

Study Design and Concept - C.H., A.H., D.K., V.H.F.

Data Gathering - Y.N., A.G.L., E.C., S.H.H.

Analysis - C.H., V.H.F, A.C.

Writing and Review of Manuscript - All authors

The Influenza in Transplant Study Group: Pilar Perez-Romero, Hospital Universitario Virgen del Rocío and Biomedicine Research Institute, Seville, Spanish Network for Research in Infectious Diseases (REIPI), Spain; Teresa Aydillo, Hospital Universitario Virgen del Rocío and Biomedicine Research Institute, Seville, Spanish Network for Research in Infectious Diseases (REIPI), Spain; Jordi Carratala, University Hospital of Bellvitge, L'Hospitalet de Llobregat, Barcelona, Spanish Network for Research in Infectious Diseases (REIPI), Spain; Patricia Munoz, 
Gregorio Marañón University Hospital, Instituto de Investigación Sanitaria Hospital Gregorio Marañón, CIBER Enfermedades Respiratorias-CIBERES, and Department of Medicine, School of Medicine, Universidad Complutense de Madrid, Madrid, Spain; Miguel Montejo, Cruces University Hospital, Vizcaya, Spanish Network for Research in Infectious Diseases (REIPI), Spain; Francisco Lopez-Medrano, University Hospital 12 de Octubre, Instituto de Investigación Biomédica i+12, Madrid, Spanish Network for Research in Infectious Diseases (REIPI), Spain; Maria Carmen Farinas, University Hospital Marqués de Valdecilla, Santander, Spanish Network for Research in Infectious Diseases (REIPI), Spain; Joan Gavalda, Vall d'Hebron University Hospital, Barcelona, Spanish Network for Research in Infectious Diseases (REIPI), Spain; Asuncion Moreno, University Clinic Hospital, Barcelona, Spanish Network for Research in Infectious Diseases (REIPI), Spain; Jesus Fortun, University Hospital Ramón y Cajal, Madrid, Spanish Network for Research in Infectious Diseases (REIPI), Spain; Julian Torre-Cisneros, Maimonides Biomedical Research Institute of Cordoba, Reina Sofia University Hospital, University of Cordoba, Córdoba, Spanish Network for Research in Infectious Diseases (REIPI), Spain; Emily Blumberg, University of Pennsylvania, USA; Lara Danziger-Isakov, Cincinnati Children's Hospital, USA; Ajit Limaye, University of Washington, USA; Marilyn Levi, University of Colorado, USA; Peter Chin-Hong and Catherine Liu, University of California, San Francisco, USA; Tanvi Sharma, Boston Children's Hospital, USA; Janet Englund, Seattle Children's Hospital, USA; Gail Reid, University of Illinois, USA; Fernanda Silveira, University of Pittsburgh, USA; Shahid Husain, University of Toronto, Canada 


\section{FIGURE LEGENDS}

Figure 1 Antibody response to influenza infection and vaccination.

Figure 1A: IgG response to infection or vaccination. H1N1 infected patients $(n=40)$ had a significant response against 27.9\% (24/86) antigens (18/24 were H1N1 antigens). H3N2 infected $(\mathrm{n}=40)$ patients responded to $43.0 \%(37 / 86)$ antigens (31/37 were H3N2 antigens). Vaccinated patients $(\mathrm{n}=40)$ had a significant response against 7.0\% $(6 / 86)$ antigens $(5 / 6$ were H1N1 antigens). Figure 1B: IgA response to infection or vaccination. H1N1 infected patients $(n=40)$ had a significant response against 23.3\% (20/86) antigens (16/20 were H1N1 antigens). H3N2 infected $(\mathrm{n}=40)$ responded to $37.2 \%(32 / 86)$ antigens $(27 / 32$ were H3N2 antigens). Vaccinated $(\mathrm{n}=40)$ had a significant response against 5.8\% (5/86) antigens (5/5 were H1N1 antigens). Antigen reactivities with a q-value of $<0.01$ and a fold-change of $\geq 2$ (4-week sample vs. baseline) were considered to be significant.

Figure 2 Heatmap showing IgG immune responses to influenza A/H1N1 antigens for H1N1 infected patients and vaccinated patients.

Influenza A/H1N1infected and vaccinated patients are indicated in red and black, respectively. Blue dots indicate H1N1 infected patients who got an influenza vaccine prior to infection within the same influenza season. Significant differences between infected and vaccinated patients were detected with the SAM algorithm with q value $<0.01$. Hierarchical clustering (using Manhattan distance) shows three major clusters: a cluster of high-responders on the left (all of them with H1N1 infection) a cluster of low responders (mixed H1N1 infection and vaccination) and a cluster with intermediate responders (mixed H1N1 infection and vaccination). Scale represents $\log 2$ of fold change as shown. HA, hemagglutinin. HA1, HA1 subunit of hemagglutinin. NP, nucleoprotein.

Figure 3 Heatmap showing IgG immune responses to influenza A/H3N2 antigens for H3N2 infected patients and vaccinated patients.

Influenza A/H3N2 infected and vaccinated patients are indicated in red and black, respectively. Blue dots indicate H3N2 infected patients who got an influenza vaccine prior to infection within the same influenza season. Significant differences between infected and vaccinated patients were detected with the SAM algorithm with q value $<0.01$. Hierarchical clustering (using Manhattan distance) shows two major clusters: a cluster of high-responders on the right, and a cluster of low 
responders at the left. Scale represents $\log 2$ of fold change as shown. HA, hemagglutinin. HA1, HA1 subunit of hemagglutinin. HAdTM, hemagglutinin without stem region. WP, whole viral particle.

This article is protected by copyright. All rights reserved 


\section{REFERENCES}

1. Kumar D, Ferreira VH, Blumberg E, et al. A Five-year Prospective Multi-center Evaluation of Influenza Infection in Transplant Recipients. Clinical infectious diseases : an official publication of the Infectious Diseases Society of America. 2018.

2. Kumar D, Michaels MG, Morris MI, et al. Outcomes from pandemic influenza A H1N1 infection in recipients of solid-organ transplants: a multicentre cohort study. The Lancet infectious diseases. 2010;10(8):521-526.

3. Kumagai T, Nakayama T, Okuno Y, et al. Humoral immune response to influenza A(H1N1)pdm2009 in patients with natural infection and in vaccine recipients in the 2009 pandemic. Viral immunology. 2014;27(8):368-374.

4. Chen MI, Barr IG, Koh GC, et al. Serological response in RT-PCR confirmed H1N1-2009 influenza a by hemagglutination inhibition and virus neutralization assays: an observational study. PloS one. 2010;5(8):e12474.

5. Esposito S, Daleno C, Tagliabue C, et al. Antibody response of healthy children to pandemic A/H1N1/2009 influenza virus. Virology journal. 2011;8:563.

6. Lerdsamran H, Pittayawonganon C, Pooruk P, et al. Serological response to the 2009 pandemic influenza A (H1N1) virus for disease diagnosis and estimating the infection rate in Thai population. PloS one. 2011;6(1):e16164.

7. Couch RB, Atmar RL, Keitel WA, et al. Randomized comparative study of the serum antihemagglutinin and antineuraminidase antibody responses to six licensed trivalent influenza vaccines. Vaccine. 2012;31(1):190-195.

8. Hirzel C, Ferreira VH, L'Huillier AG, et al. Humoral response to natural influenza infection in solid organ transplant recipients. American journal of transplantation : official journal of the American Society of Transplantation and the American Society of Transplant Surgeons. 2019;19(8):23182328 .

9. Hirzel C, Kumar D. Influenza vaccine strategies for solid organ transplant recipients. Current opinion in infectious diseases. 2018.

10. Krammer F, Smith GJD, Fouchier RAM, et al. Influenza. Nature reviews Disease primers. 2018;4(1):3.

11. Kaminski DA, Lee FE. Antibodies against conserved antigens provide opportunities for reform in influenza vaccine design. Front Immunol. 2011;2:76.

12. Paterson D, Fodor E. Emerging roles for the influenza A virus nuclear export protein (NEP). PLoS pathogens. 2012;8(12):e1003019.

13. Hale BG, Randall RE, Ortin J, Jackson D. The multifunctional NS1 protein of influenza A viruses. The Journal of general virology. 2008;89(Pt 10):2359-2376. 
14. Berthoud TK, Hamill M, Lillie PJ, et al. Potent CD8+ T-cell immunogenicity in humans of a novel heterosubtypic influenza A vaccine, MVA-NP+M1. Clinical infectious diseases : an official publication of the Infectious Diseases Society of America. 2011;52(1):1-7.

15. Gabbard J, Velappan N, Di Niro R, et al. A humanized anti-M2 scFv shows protective in vitro activity against influenza. Protein engineering, design \& selection : PEDS. 2009;22(3):189-198.

16. Memoli MJ, Shaw PA, Han A, et al. Evaluation of Antihemagglutinin and Antineuraminidase Antibodies as Correlates of Protection in an Influenza A/H1N1 Virus Healthy Human Challenge Model. mBio. 2016;7(2):e00417-00416.

17. Koopmans M, de Bruin E, Godeke GJ, et al. Profiling of humoral immune responses to influenza viruses by using protein microarray. Clinical microbiology and infection : the official publication of the European Society of Clinical Microbiology and Infectious Diseases. 2012;18(8):797-807.

18. Huijskens EG, Reimerink J, Mulder PG, et al. Profiling of humoral response to influenza A(H1N1)pdm09 infection and vaccination measured by a protein microarray in persons with and without history of seasonal vaccination. PloS one. 2013;8(1):e54890.

19. Desbien AL, Van Hoeven N, Reed SJ, et al. Development of a high density hemagglutinin protein microarray to determine the breadth of influenza antibody responses. BioTechniques. 2013;54(6):345-348.

20. Price JV, Jarrell JA, Furman D, et al. Characterization of influenza vaccine immunogenicity using influenza antigen microarrays. PloS one. 2013;8(5):e64555.

21. Wumkes ML, van der Velden AM, de Bruin E, et al. Microarray profile of the humoral immune response to influenza vaccination in breast cancer patients treated with chemotherapy. Vaccine. 2017;35(9):1299-1305.

22. Nakajima R, Supnet M, Jasinskas A, et al. Protein Microarray Analysis of the Specificity and Cross-Reactivity of Influenza Virus Hemagglutinin-Specific Antibodies. mSphere. 2018;3(6).

23. Natori Y, Shiotsuka M, Slomovic J, et al. A Double-Blind, Randomized Trial of High-Dose vs Standard-Dose Influenza Vaccine in Adult Solid-Organ Transplant Recipients. Clinical infectious diseases : an official publication of the Infectious Diseases Society of America. 2018;66(11):16981704.

24. Chruscinski A, Huang FY, Nguyen A, et al. Generation of Antigen Microarrays to Screen for Autoantibodies in Heart Failure and Heart Transplantation. PLoS One. 2016;11(3):e0151224.

25. Chruscinski A, Huang FY, Ulndreaj A, et al. Generation of Two-color Antigen Microarrays for the Simultaneous Detection of IgG and IgM Autoantibodies. Journal of visualized experiments : JoVE. 2016(115).

26. Grosman-Rimon L, Ajrawat P, Lioe J, et al. Increases in Serum Autoantibodies After Left Ventricular Assist Device Implantation. Journal of cardiac failure. 2019.

This article is protected by copyright. All rights reserved 
27. Miller E, Hoschler K, Hardelid P, Stanford E, Andrews N, Zambon M. Incidence of 2009 pandemic influenza A H1N1 infection in England: a cross-sectional serological study. Lancet. 2010;375(9720):1100-1108.

28. Tusher VG, Tibshirani R, Chu G. Significance analysis of microarrays applied to the ionizing radiation response. Proceedings of the National Academy of Sciences of the United States of America. 2001;98(9):5116-5121.

29. Eisen MB, Spellman PT, Brown PO, Botstein D. Cluster analysis and display of genome-wide expression patterns. Proceedings of the National Academy of Sciences of the United States of America. 1998;95(25):14863-14868.

30. Krammer F. The human antibody response to influenza A virus infection and vaccination. Nature reviews Immunology. 2019;19(6):383-397.

31. Monto AS, Malosh RE, Petrie JG, Martin ET. The Doctrine of Original Antigenic Sin: Separating Good From Evil. The Journal of infectious diseases. 2017;215(12):1782-1788.

32. Li Y, Myers JL, Bostick DL, et al. Immune history shapes specificity of pandemic H1N1 influenza antibody responses. The Journal of experimental medicine. 2013;210(8):1493-1500.

33. Rajendran M, Nachbagauer R, Ermler ME, et al. Analysis of Anti-Influenza Virus Neuraminidase Antibodies in Children, Adults, and the Elderly by ELISA and Enzyme Inhibition: Evidence for Original Antigenic Sin. mBio. 2017;8(2).

34. de Boer GF, Back W, Osterhaus AD. An ELISA for detection of antibodies against influenza A nucleoprotein in humans and various animal species. Archives of virology. 1990;115(1-2):47-61.

35. Sukeno N, Otsuki Y, Konno J, et al. Anti-nucleoprotein antibody response in influenza A infection. The Tohoku journal of experimental medicine. 1979;128(3):241-249.

36. Verma N, Dimitrova M, Carter DM, et al. Influenza virus H1N1pdm09 infections in the young and old: evidence of greater antibody diversity and affinity for the hemagglutinin globular head domain (HA1 Domain) in the elderly than in young adults and children. Journal of virology. 2012;86(10):5515-5522.

37. Haaheim R. Single-radial-complement-fixation: a new immunodiffusion technique. 2. Assay of the antibody response to the internal antigens (MP and NP) of influenza A virus in human sera after vaccination and infection. Developments in biological standardization. 1977;39:481-484.

38. Cox RJ, Brokstad KA. The postvaccination antibody response to influenza virus proteins. APMIS : acta pathologica, microbiologica, et immunologica Scandinavica. 1999;107(3):289-296.

39. Monto AS, Petrie JG, Cross RT, et al. Antibody to Influenza Virus Neuraminidase: An Independent Correlate of Protection. The Journal of infectious diseases. 2015;212(8):1191-1199.

This article is protected by copyright. All rights reserved 
40. Natori Y, Shiotsuka M, Slomovic J, et al. A Double Blind Randomized Trial of High Dose vs. Standard Dose Influenza Vaccine in Adult Solid Organ Transplant Recipients. Clinical infectious diseases : an official publication of the Infectious Diseases Society of America. 2017.

41. Schroeder HW, Jr., Cavacini L. Structure and function of immunoglobulins. The Journal of allergy and clinical immunology. 2010;125(2 Suppl 2):S41-52.

42. Li ZN, Lin SC, Carney PJ, et al. IgM, IgG, and IgA antibody responses to influenza A(H1N1)pdm09 hemagglutinin in infected persons during the first wave of the 2009 pandemic in the United States. Clinical and vaccine immunology : CVI. 2014;21(8):1054-1060.

43. Beyer WE, Van der Logt JT, van Beek R, Masurel N. Immunoglobulin G, A and M response to influenza vaccination in different age groups: effects of priming and boosting. The Journal of hygiene. 1986;96(3):513-522.

44. Gould VMW, Francis JN, Anderson KJ, Georges B, Cope AV, Tregoning JS. Nasal IgA Provides Protection against Human Influenza Challenge in Volunteers with Low Serum Influenza Antibody Titre. Frontiers in microbiology. 2017;8:900.

45. Rosenberg JM, Utz PJ. Protein microarrays: a new tool for the study of autoantibodies in immunodeficiency. Frontiers in immunology. 2015;6:138.

46. Haddon DJ, Diep VK, Price JV, Limb C, Utz PJ, Balboni I. Autoantigen microarrays reveal autoantibodies associated with proliferative nephritis and active disease in pediatric systemic lupus erythematosus. Arthritis Res Ther. 2015;17:162.

\section{SUPPORTING INFORMATION}

Additional supporting information may be found online in the Supporting Information section at the end of the article. 
Table 1 Demographics of influenza A virus infected and influenza vaccinated cohort.

\begin{tabular}{|c|c|c|c|c|}
\hline Characteristic & All $(n=120)$ & $\begin{array}{l}\text { Influenza } A \\
\text { infected }(n=80)\end{array}$ & $\begin{array}{l}\text { Influenza } \\
\text { vaccinated } \\
(\mathrm{n}=40)\end{array}$ & $\begin{array}{l}\mathrm{P} \\
\text { value }\end{array}$ \\
\hline Age (years), median (IQR) & $55.5(45-63)$ & $55(44.5-65)$ & $57(49.5-62.5)$ & 0.911 \\
\hline Male sex & $82(68.3 \%)$ & $51(63.8 \%)$ & $31(77.5 \%)$ & 0.127 \\
\hline $\begin{array}{l}\text { Time from transplantation to } \\
\text { infection/vaccination, years } \\
\text { (IQR) }\end{array}$ & $3.0(0.8-8.1)$ & $2.8(0.4-9.6)$ & $3.3(1.0-7.4)$ & 0.515 \\
\hline $\begin{array}{l}\text { Within one year of } \\
\text { transplantation }\end{array}$ & $39(32.5 \%)$ & $28(35.0 \%)$ & $11(27.5 \%)$ & 0.408 \\
\hline \multicolumn{5}{|l|}{ Type of transplant } \\
\hline Kidney & $58(48.3 \%)$ & $46(57.5 \%)$ & $12(30.0 \%)$ & 0.006 \\
\hline Liver & $25(20.8 \%)$ & $16(20.0 \%)$ & $9(22.5 \%)$ & 0.813 \\
\hline Lung & $17(14.2 \%)$ & $9(11.3 \%)$ & $8(20.0 \%)$ & 0.266 \\
\hline Heart & $10(8.3 \%)$ & $4(5.0 \%)$ & $6(15.0 \%)$ & 0.082 \\
\hline Intestine & $1(0.8 \%)$ & $1(1.3 \%)$ & $0(0.0 \%)$ & 1.000 \\
\hline Combined & $9(7.5 \%)$ & $4(5.0 \%)$ & $5(12.5 \%)$ & 0.185 \\
\hline \multicolumn{5}{|l|}{ Immunosuppression } \\
\hline Prednisone & $93(77.5 \%)$ & $64(80.0 \%)$ & $29(72.5 \%)$ & 0.354 \\
\hline $\begin{array}{l}\text { Prednisone dose, } \\
\text { mg/day (IQR) }\end{array}$ & $5(5-10)$ & $5(5-10)$ & $5(3.8-8.8)$ & 0.532 \\
\hline Tacrolimus & $77(64.2 \%)$ & $49(61.3 \%)$ & $28(70.0 \%)$ & 0.346 \\
\hline Cyclosporine & $37(30.8 \%)$ & $26(32.5 \%)$ & $11(27.5 \%)$ & 0.576 \\
\hline $\begin{array}{l}\text { Mycophenolate } \\
\text { mofetil/mycophenola } \\
\text { te sodium }\end{array}$ & $88(73.3 \%)$ & $60(75.0 \%)$ & $32(80.0 \%)$ & 0.559 \\
\hline Azathioprine & $8(6.7 \%)$ & $3(3.8 \%)$ & $5(12.5 \%)$ & 0.070 \\
\hline Sirolimus & $11(9.2 \%)$ & $10(12.5 \%)$ & $1(2.5 \%)$ & 0.074 \\
\hline $\begin{array}{l}\text { Antithymocyte globulin } \\
\text { within } 6 \text { months prior }\end{array}$ & $6(5.1 \%)$ & $5(6.4 \%)$ & $1(2.5 \%)$ & 0.662 \\
\hline $\begin{array}{l}\text { Rejection within } 6 \text { months } \\
\text { prior }\end{array}$ & $4(3.3 \%)$ & $4(5.0 \%)$ & $0(0.0 \%)$ & 0.300 \\
\hline
\end{tabular}

IQR, interquantile range. $\mathrm{mg}$, milligram. 


\section{Figure 1}

A

IgG antibodies

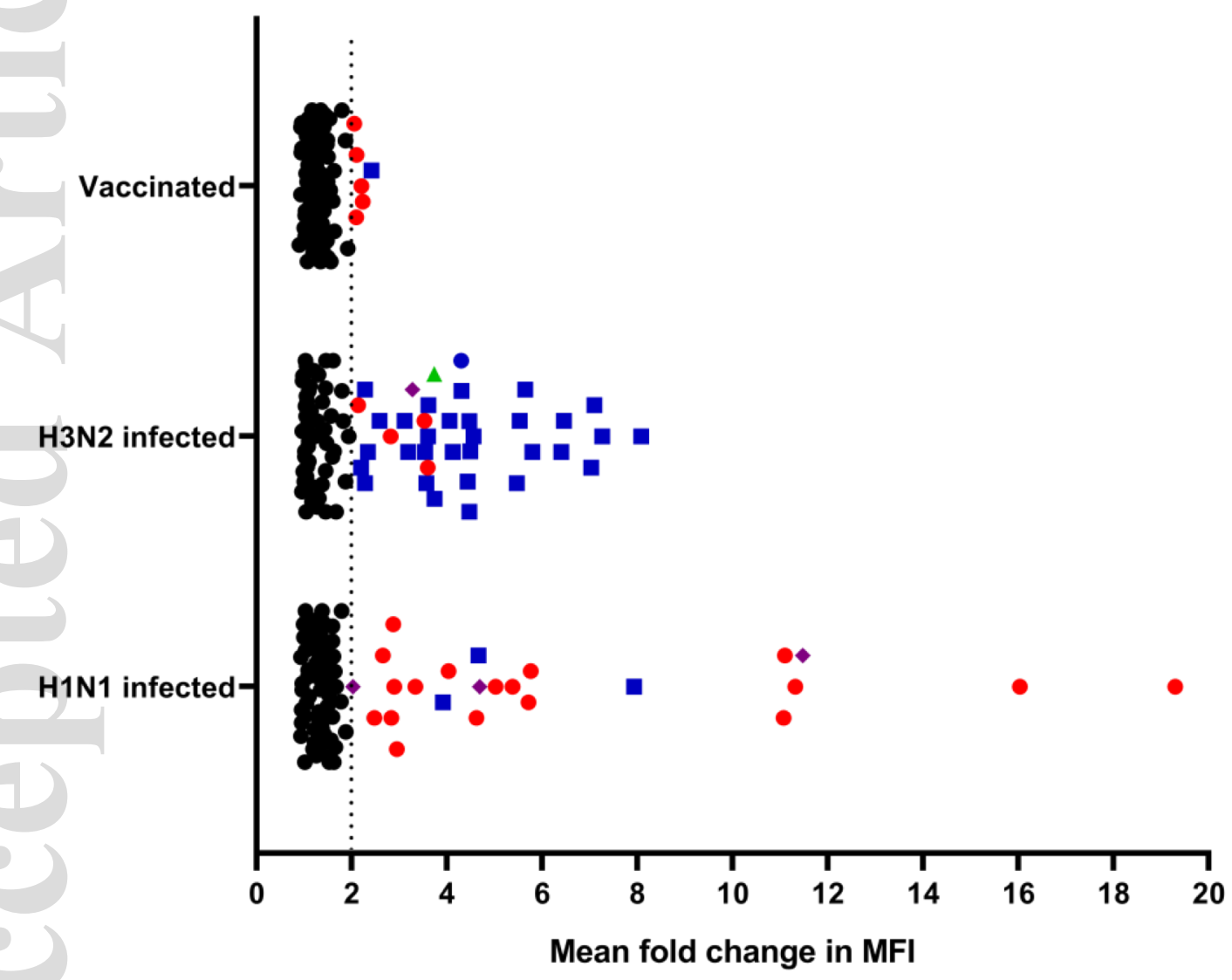

- Antigens without significant response

- H1N1 antigen with significant response
B

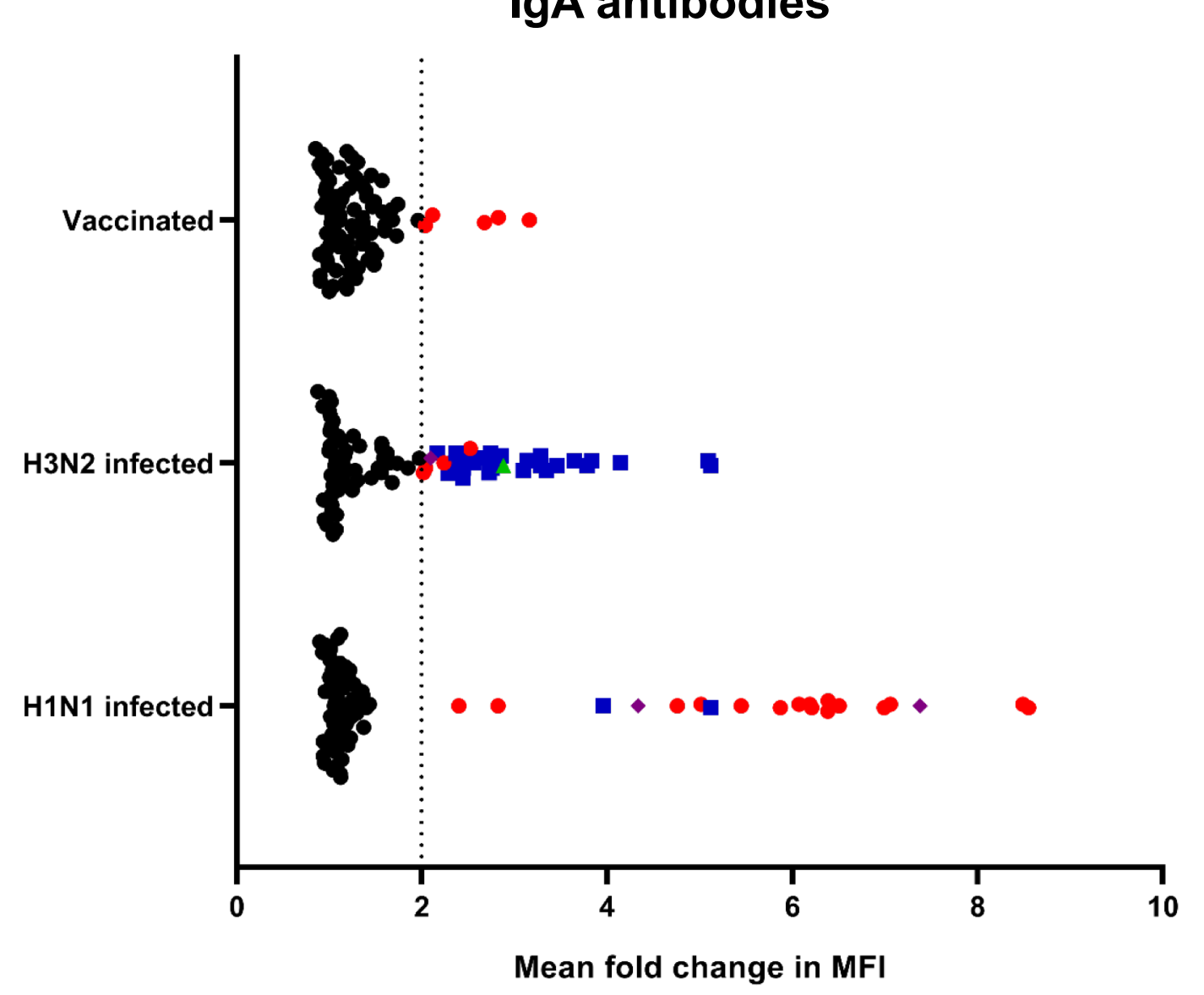

- H3N2 antigen with significant response

H5N1 antigen with significant response $\Delta B$ antigen with significant response 


\section{Figure 2}

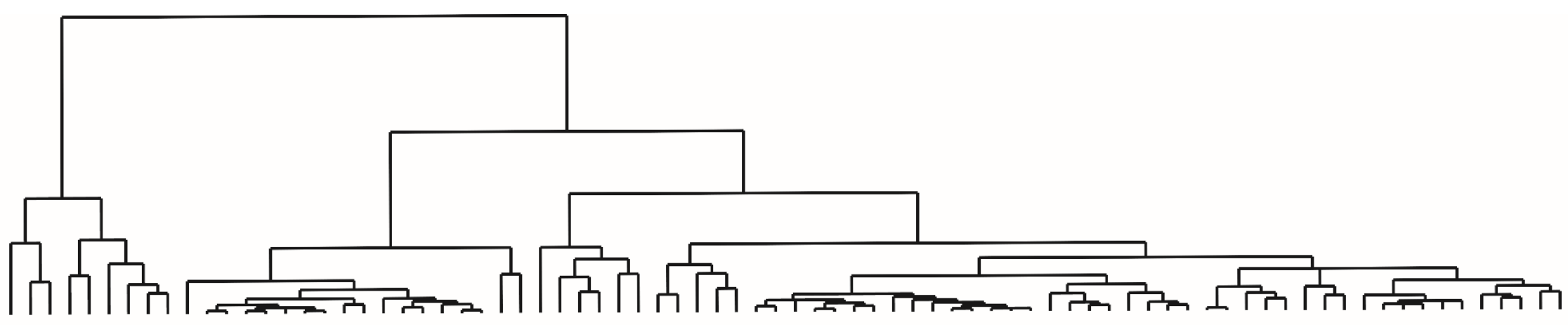

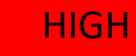
LOW INTERMEDIATE

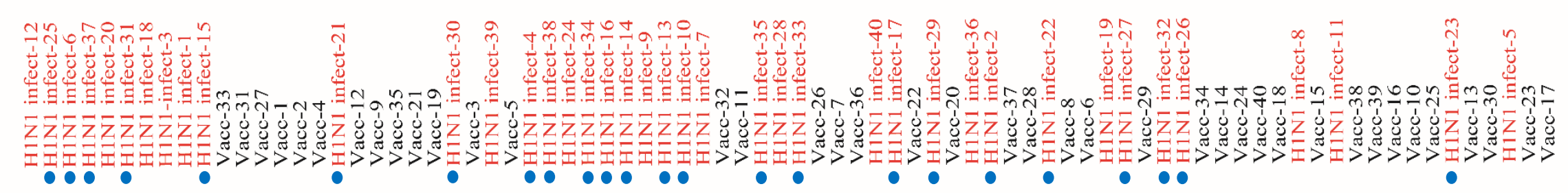

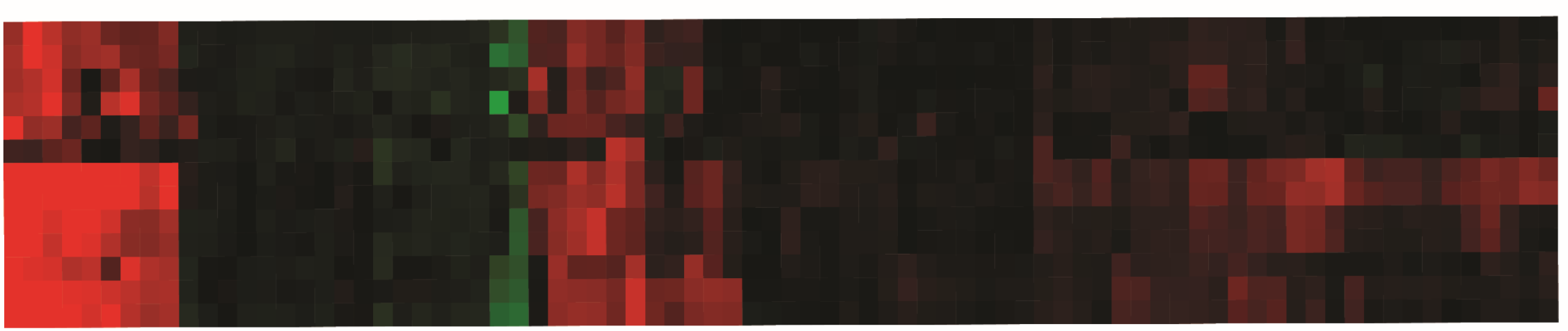

H1N1 A/New Caledonia/20/1999 - HA HIN1 A/Solomon Islands/3/2006 - HA HIN1 A/New Caledonia/20/1999 - HA1 H1N1 A/Solomon Islands/3/2006 - HA H1N1 A/California/07/2009 - NP H1N1 A/06/California/2009 - NP H1N1 A/Texas/05/2009 - HA1 H1N1 A/England/195/2009 - HAl H1N1 A/Texas/05/2009 - HA H1N1 A/England/195/2009 - HA HINl A/California/07/2009-pdm09 - HA H1N1 A/New York/18/2009 - HA 
Figure 3
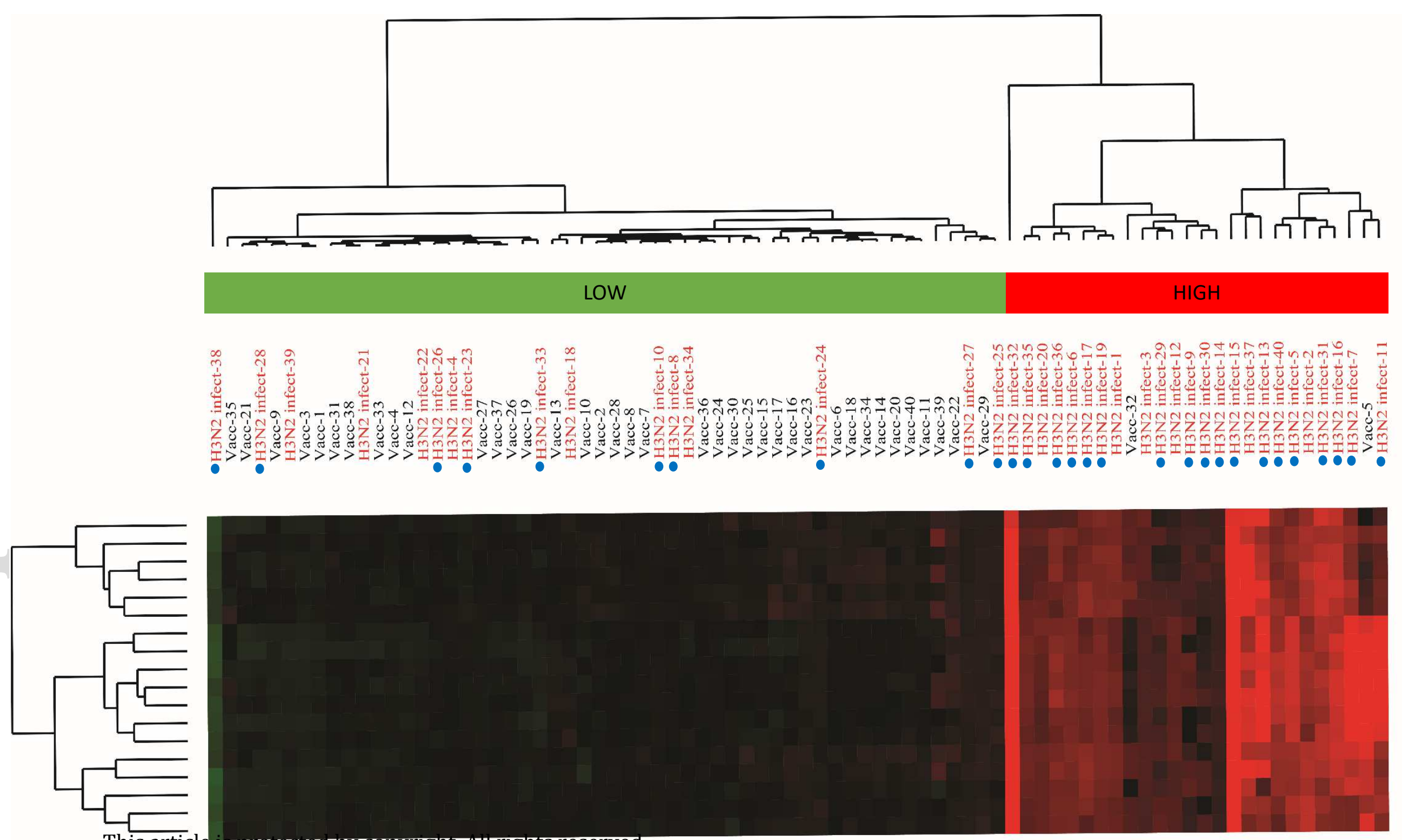

H3N2 A/California/7/2004 - HA H3N2 A/Brisbane/10/2007 - HA H3N2 A/Wisconsin/677/2005 - HA H3N2 A/Uruguay/716/2007 - HA HNN2 AFrujlan/41/2002- HA

H3N2 A/Hiroshima/52/2005 - HA

H3N2 A/Texas/50/2012 - HAl

H3N2 A/Victoria/210/2009 - HAI

H3N2 A/Victoria/361/2011 - HA1

(3)

H3N2 A/ndianana $72012-\mathrm{H} A$

H3N2 Alowa 08 2011-

H3N2 A/Switzerland/97/15293/2013 - HA1

H3N2 A/Caledonia/71/2014- HA dTM

H3N2 A/Wisconsin'15/2009 - HA dTM 